\title{
FOURIER CORRELATION ANALYSIS OF GC-SPECTRA OF SHEEP MILK AND DAIRY
}

\author{
HILMA, E[lena]; MIERLITA, D[an] \& TEUSDEA, A[lin] C[ristian]
}

\begin{abstract}
Authors present the discrimination performances of amplitude and phase-only Fourier correlation over the "twin" typed GC-spectra of sheep milk and ripened cheese. Therefore are built up the correlation matrix over the 12 analysed GCspectra in both the amplitude and phase domains, in order to assess the most robust Fourier correlation method for the "twin" GC-spectra discrimination.
\end{abstract}

Key words: phase-only, Fourier correlation, GC-spectra, sheep milk.

\section{INTRODUCTION}

One way to measure the discrimination of "twin" type samples is the Fourier correlation analysis. Such a method is Fourier correlation (FC) with the classical process in the amplitude domain (AmC). The amplitude domain Fourier correlation doesn't counts the variation speed (i.e. the first order derivative) of the analysed functions and thus for "twin" functions can fail their discrimination. Phase-only Fourier correlation (POC) (Chien, 2004; Ito et al., 2004; Miyazawa et al., 2005) as it is named takes into account the phase or the variation speed of amplitude values of neighbouring points from the profile data. Theoretically it can discriminate better the "twin" functions. The present paperwork analyse the discrimination performances of the (AmC) and (POC) over the "twin" type spectra. Spectra database used consists of "twin" gas-chromatogram (GC) spectra. The GC-spectra were built up in food processing experiment with sheep milk and dairy.

\section{PATTERN RECOGNITION CORRELATION METHODS}

Fourier correlation process is used for function discrimination. That means one function is "compared" with several ones. Comparison criteria concludes if the compared functions are or not similar each other. The comparison process works basically with two functions at the same time. In our case the comparison method is the cross-correlation and the functions are the GC-spectra. In a single cross-correlation process the two functions are denoted as reference and nonreference.

The classical amplitude cross-correlation (AmC) considers two (N-size) GC-spectra: $\operatorname{ref}(x)$ as reference GC-spectrum and $\operatorname{nref}(x)$ as non-reference GC-spectrum. The discrete Fourier transforms of these GC-spectra, denoted as $\operatorname{Ref}(u)$ and $N R e f(u)$, are given by (Teusdea, 2009)

$$
\begin{gathered}
\operatorname{Ref}(u)=\operatorname{REF}(u) \cdot e^{i \varphi_{\text {ref }}(u)} \\
\operatorname{NRef}(u)=\operatorname{NREF}(u) \cdot e^{i \varphi_{\text {rref }}(u)} \\
\operatorname{REF}(u)=[\operatorname{Ref}(u) \cdot \overline{\overline{\operatorname{Ref}(u)}}]^{0.5} \\
\operatorname{NREF}(u)=[\operatorname{NRef}(u) \cdot N \overline{\overline{\operatorname{Ref}(u)}}]^{0.5},
\end{gathered}
$$

where $\operatorname{REF}(u)$ and $\operatorname{NREF}(u)$ are the amplitude part, $\varphi_{\text {ref }}(u)$ and $\varphi_{\text {nref }}(u)$, are the phase part of the Fourier transforms.

Classical correlation function, $\operatorname{ClC}_{R N}(x)$, is the inverse discrete Fourier transform of classical cross-spectrum, given by

$$
\operatorname{ClC}_{R N}(x)=\frac{1}{N} \sum_{u=1}^{N}\left[R E F(u) \cdot N R E F(u) \cdot e^{i \Delta \varphi_{r n}(u)} \cdot e^{-\frac{2 \pi i}{N} u x}\right]
$$

where the $\Delta \varphi_{r n}(u)$ is the phase difference between the reference and non-reference function. This function presents a highly wide cross-correlation peak when $\operatorname{ref}(x)=\operatorname{nre} f(x)$. When $\operatorname{ref}(x) \neq \operatorname{nref}(x)$ then the cross-correlation peak strongly decreases.

The phase-only cross-spectrum is defined by (Ito et al., 2004; Miyazawa et al., 2006)

$$
P O \_C S=\frac{R E F(u) \cdot N R E F(u) \cdot e^{i \Delta \varphi_{r n}(u)}}{\left|\operatorname{REF}(u) \cdot N R E F(u) \cdot e^{i \Delta \varphi_{r n}(u)}\right|}=e^{i \Delta \varphi_{r n}(u)}
$$

and thus the phase-only cross-correlation is given by Fourier transform of the phase-only cross-spectrum (Teusdea, 2009)

$$
\operatorname{POC}(x)=\frac{1}{N} \sum_{u=1}^{N}\left[e^{i \Delta \varphi_{r n}(u)} \cdot e^{-\frac{2 \pi i}{N} u x}\right]
$$

If $\operatorname{ref}(x)=\operatorname{nref}(x)$ then $\Delta \varphi_{r n}(u)=0$ and the phase only correlation is give by (Teusdea, 2009)

$$
\operatorname{POC}(x)=\frac{1}{N} \sum_{u=1}^{N}\left[1 \cdot e^{-\frac{2 \pi i}{N} u x} \cdot e^{-\frac{2 \pi i}{M} v y}\right]=\delta(x) .
$$

This means that if the two GC-spectra are identical then the POC gives a highly sharp peak so the matching accuracy is higher than in the classical method.

Better understanding and symmetry with first order statistical correlation coefficient, was used the normalized Fourier Correlation coefficient NFCC given by (Teusdea, 2009)

$\operatorname{NFCC}(f(x), g(x))=$

$\max _{x}\left[\frac{\left[\mathcal{F}^{-1}[\overline{F(-u)} \cdot G(u)]\right](x)}{\max _{x}\left[\left[\mathcal{F}^{-1}\left[|F(u)|^{2}\right]\right](x)\right] \cdot \max _{x}\left[\left[\mathcal{F}^{-1}\left[|G(u)|^{2}\right]\right](x)\right]}\right]$

where $\mathcal{F}^{-1}$ is the inverse Fourier transform operator, $F(u)$ and $G(u)$ are the Fourier transform of the $f(x)$ and $g(x)$ functions. If these Fourier transforms are replaced with the phase-only part of them then the NFCC becomes PhNFCC, otherwise is denoted as AmNFCC.

\section{RESULTS AND DISCUSSION}

In the sheep dairy benchmark experiment were considered samples of fresh sheep milk without (M0) and with $0.05 \%$ (M005), $0.10 \%$ (M010), 0.15\% (M015) fish oil additive. The 
fish oil is added to improve the fatty acid content of the milk and thus mainly to gain the $\omega_{3}$ and $\omega_{6}$ content with cardiological and neurogical benefits for the human body (Sofi et al., 2010).

After the milk preparation were processed samples of ripened spun paste cheese (SPC0, SPC005, SPC010, SPC015) and ripened Teleme cheese (TC0, TC005, TC010, TC015). The main goal of the experiment was to analyse the fatty acid profiles from the 12 mentioned samples. Hence were done the $\mathrm{GC}$-spectra of these samples. The GC-spectra were very similar or "twin" spectra because, from the fatty acids profile point of view, the analysed samples were statistically strong-correlated.

As mentioned above one accurate way to discriminate the "twin profile" data is the Fourier correlation in the amplitude domain (eqn. 1-5) and phase domain (eqn. 6-8).

Correlation matrix between the $12 \mathrm{GC}$-spectra of the milk, spun paste cheese and Teleme cheese were built for amplitude correlation coefficient AmNFCC (figure 1) and phase-only correlation coefficient PhNFCC (figure 2). The correlation values were built up for several Fourier vector size $2^{m}, m=\overline{1,20}$, and then were calculated theirs $L^{p}$ norm $(p=2.5)$.

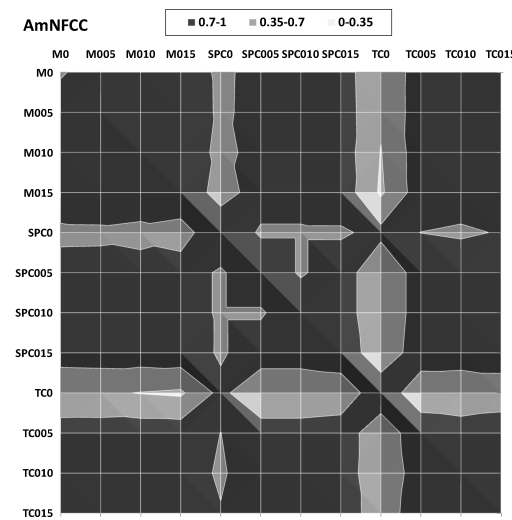

Fig. 1. Amplitude correlation matrix of GC-spectra.

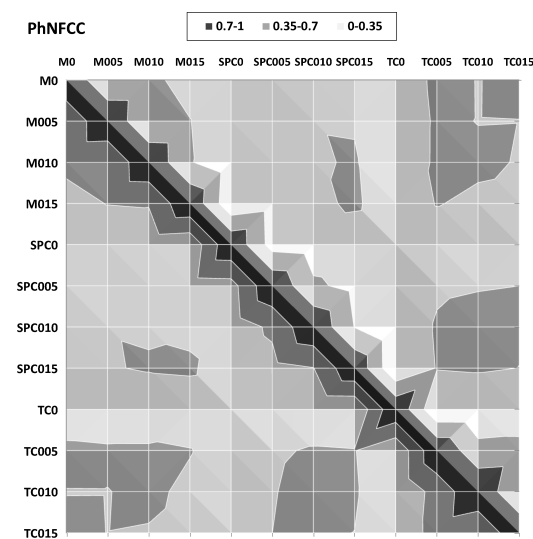

Fig. 2. Phase-only correlation matrix of GC-spectra.

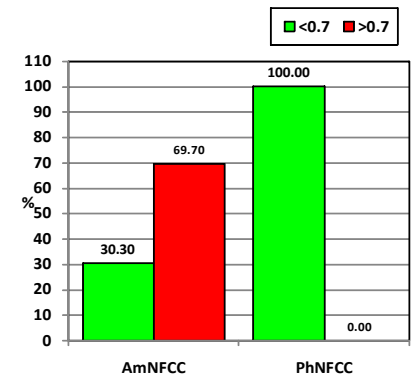

Fig. 3. Comparison of the statistical significance of the correlation matrix values ("twin" discrimination statistical threshold is $\leq 0.7$ ).
This algorithm increases the accuracy of the correlation process. The statistical significance of the AmNFCC and PhNFCC is: $0-0.35$ strong discrimination, 0.35-0.70 good discrimination and $0.70-1.00$ poor discrimination.

As it showed in figure 1 the AmNFCC has poor discrimination of "twin" GC-spectra as the correlation matrix graph is dark coloured. Figure 2 shows that the PhNFCC has strong discrimination of "twin" GC-spectra as the correlation matrix graph is light coloured in the non-diagonal area.

Figure 3 presents the percentage of discrimination values for AmNFCC and PhNFCC. It is revealed that only in 30.30\% cases the amplitude Fourier correlation do not fail to discriminate the "twin" type analysed GC-spectra. In the same way the phase-only Fourier correlation has a $100 \%$ of discrimination of "twin" type analysed GC-spectra.

\section{CONCLUSION}

In this paper, there are presented the Fourier correlations in amplitude (AmNFCC) and phase-only (PhNFCC) domain for discrimination the "twin" type GC-spectra of sheep milk, ripened spun paste cheese and ripened Teleme cheese. The results in figures 1-3 emphasizes that just the phase-only Fourier correlation (PhNFCC) has 100\% discrimination performances on "twin" type GC-spectra. The Fourier correlation in the amplitude domain (AmNFCC) fails in $69.70 \%$ to discriminate the same "twin" GC-spectra.

Our future plan is to work with much larger "twin" GCspectra database to ensure higher statistical significance.

\section{ACKNOWLEDGEMENTS}

This work was supported by CNCSIS-UEFISCSU, Romanian project number PNII-IDEI, ID-679/2008, number 1082/2009.

\section{REFERENCES}

Chien, L. H. \& Aoki T.(2004). Robust motion estimation for video sequences based on phase-only correlation, Proceedings of the 6th IASTED SIP 2004, Hamza M.H. (Ed), pp. 441-446, ISBN 0-88986-434-9, Honolulu, USA, Aug. 2004, ACTA Press, Canada.

Ito , K.; Nakajima H.; Kobayashi K. \& Aoki T., Higuchi T. (2004). A fingerprint matching algorithm using phaseonly correlation, IEICE Transactions. Fundamentals, E87-A, No. 3, March 2004, pp. 682-691, ISSN 17451337.

Miyazawa K.; Ito K.; Aoki T.; K. Kobayashi \& Nakajima H.a (ICB 2006), A phase-based iris recognition algorithm, In: Lecture Notes in Computer Science 3832, D. Zhang and A.K. Jain (Ed.), ISBN 3-540-3111-4,pp. 356-365, Springer-Verlag Berlin Heidelberg Germany.

Sofi F.; Buccioni A.; Cesari F.; Gori A.M.; Minieri S.; Mannini L.; Casini A.; Gensini G.F.; Abbate R., Antongiovanni M. (2010), Effects of a dairy product (pecorino cheese) naturally rich in cis-9, trans-11 conjugated linoleic acid on lipid, inflammatory and haemorheological variables: A dietary intervention study, Nutrition, Metabolism and Cardiovascular Diseases, Volume 20, Issue 2, February Pages 117-124.

Teusdea, A.C. \& Gabor, G. (2009). Iris Recognition with Phase-Only Correlation, Annals of DAAAM for 2009 \& Proceedings of the 20th International DAAAM Symposium, ISBN 978-3-901509-68-1, ISSN 1726-9679, pp 690-691, Editor B. Katalinic, Published by DAAAM International, Vienna, Austria. 